\title{
Automated Artifact Removal in EEG Signals of Brain Computer Interface using Wavelets and
} ICA

\author{
Rajashekhar U, Neelappa, K. Viswanath
}

\begin{abstract}
A brain-computer interface (BCI) gives a correspondence channel that interconnects the mind with an outside device. The most generally utilized system for getting BCI control signals from the brain is the electroencephalogram (EEG). In the proposed paper, BCI framework towards an EEG chronicles are reviewed into and found that the expansion of a counterfeit motion toward it, which is brought about by eye flickers, eye development, muscle and cardiovascular commotion, just as non-natural sources (e.g., control line clamor). According to the writing survey it is discovered that these issues can be overwhelmed by utilizing mix of wavelet deterioration, independent component analysis (ICA), and thresholding.

Index Terms: Automated artifacts removal, ICA, BSS, MSSA, Wavelet Transform.
\end{abstract}

\section{INTRODUCTION}

In 1929 Hans Berger find the human electroencephalogram (EEG) action [5], EEG estimations were predominantly utilized for restorative reasons or for research in the zone of cerebrum work. Over the most recent 15 years, applications have been created permitting the utilization of EEG action as a non-strong correspondence channel or as a guide in engine reclamation after loss of motion, alleged mind PC interfaces (BCIs) [5]. Cerebrum PC interface (BCI), otherwise called mind machine interface (BMI) and is a framework which empowers people to communicate with their surroundings through utilizing control sign produced from electroencephalographic (EEG) movement, without the intercession of muscles and fringe nerves. This is an immediate correspondence pathway between a human mind and an outside gadget [1]. Without requiring any development this sort of framework enables individuals to communicate through direct estimations of mind action [2]. BCI has pulled in expanding consideration of an assortment of research networks, including neuroscience, AI, recovery drug, neuroimaging, design acknowledgment, signal handling, etc.

Revised Manuscript Received on October 30, 2019.

* Correspondence Author

Rajashekhar U*, Govt. Engineering College, Haveri Karnataka, India Uraju54@gmail.com

Dr.Neelappa, Govt. Engineering College, Kushalnagar, Karnataka, India -571234 neel.m.dy@gmail.com,

Dr.K.Viswanath' Professor \& Head, Dept. of ECE, R.L.Jalappa Institute of Technology, Bangalore, vishwanathk@rljit.in

(C) The Authors. Published by Blue Eyes Intelligence Engineering and Sciences Publication (BEIESP). This is an open access article under the CC-BY-NC-ND license http://creativecommons.org/licenses/by-nc-nd/4.0/
One of the significant objectives of BCI research is to reestablish standard capacities for individuals with serious neuromuscular incapacities or upgrade certain capacities for sound individual through another sign pathway. Apparently, the most regularly utilized technique for gaining BCI control signals from the mind is the electroencephalogram (EEG). The EEG is broadly utilized in clinical practice as a result of its absence of symptoms and its minimal effort because of its noninvasive nature [3]. The EEG records include electrophysiological action created from cortical neuronal action and anticipated through the scalp and skull [6]. So in getting EEG flag there is an expansion of relics to it those might be wellspring of commotion in EEG acquisitions and they are brought about by endogenous e.g., physiological sources, for example, eye, muscle and heart movement and exogenous e.g., non-physiological sources, for example, control line coupling, impedance jumble and so forth [4].This brings down the nature of electroencephalography (EEG).Various creator presented different technique For instance, a straightforward methodology is high pass channel, Wavelet Transform, Blind source division (BSS) and Multivariate particular range investigation (MSSA) based correction[8], FASTER (Fully robotized factual thresholding), LAMIC (Lagged auto-shared data bunching) for evacuation of ancient rarities polluted in an EEG..

\section{TYPES OF BCIS}

The BCIs can be for the most part named (1) exogenous or endogenous and (2) synchronous (sign paced) or offbeat (self-managed). Kinds of BCI are recorded in Tables 2.1 and 2.2, alongside data identified with cerebrum signals which pass on data just as points of interest and drawbacks. Additionally, BCIs can be characterized into reliant and free [6]. This qualification won't be point by point in this audit since it is fundamentally the same as exogenous and endogenous refinement. Favorable circumstances and detriments in the two scientific classifications are practically equivalent. 


\section{Automated Artifact Removal in EEG Signals of Brain Computer Interface using Wavelets and ICA}

Table 1 Main differences between exogenous andendogenous BCI

\begin{tabular}{|c|c|c|c|}
\hline Approach & Brain signals & Advantages & Disadvantages \\
\hline $\begin{array}{c}\text { Endogenous } \\
\text { BCI }\end{array}$ & $\begin{array}{c}\text { - SCPs } \\
\text { - Sensorimotor } \\
\text { rhythms }\end{array}$ & $\begin{array}{c}\text { > Independent of any incitement } \\
>\text { Can be worked at choice } \\
>\text { Useful for clients with tactile } \\
\text { organs influenced } \\
>\text { Suitable for cursor control } \\
\text { applications }\end{array}$ & $\begin{array}{c}\text { Very tedious preparing (months } \\
\text { or weeks) } \\
>\text { Not all clients can get control } \\
>\text { Multichannel EEG accounts } \\
\text { equired execution } \\
>\text { Lower bit rate (20- } 30 \\
\text { bits/min) }\end{array}$ \\
\hline $\begin{array}{l}\text { Exogenous } \\
\text { BCI }\end{array}$ & $\begin{array}{l}\text { - SSVEP } \\
\text { - P300 }\end{array}$ & $\begin{array}{c}>\text { Minimal preparing } \\
>\text { Control sign set-up effectively and } \\
\text { rapidly } \\
>\text { High bit rate }(60 \text { bits/min) } \\
>\text { Only one EEG channel required }\end{array}$ & $\begin{array}{l}>\text { Permanent regard for outside } \\
\text { boosts } \\
>\text { May cause tiredness in a few } \\
\text { clients }\end{array}$ \\
\hline
\end{tabular}

Table 2 Main Differences between Synchronous And Asynchronous BCI's

\begin{tabular}{|l|l|c|}
\hline Approach & Advantages & Disadvantages \\
\hline $\begin{array}{l}\text { Synchronous } \\
\text { BCI }\end{array}$ & $\begin{array}{c}>\text { Simpler plan and execution assessment } \\
\text { The client can abstain from producing curios since } \\
\text { they can perform squints and other eye developments } \\
\text { when mind sign are not investigated }\end{array}$ & $\begin{array}{c}>\text { Does not offer a progressively } \\
\text { characteristic method } \\
\text { connection } \\
\text { of }\end{array}$ \\
\hline $\begin{array}{l}\text { Asynchronous } \\
\text { BCI }\end{array}$ & $>$ No necessity to sit tight for outside prompts \\
& $\begin{array}{c}>\text { Offers a progressively characteristic method of } \\
\text { communication }\end{array}$ & $\begin{array}{c}>\text { Increasingly } \\
\text { assessment }\end{array}$ \\
\hline
\end{tabular}

\section{TECHNIQUES FOR RECORDING OF BRAIN ACTIVITY}

Normal procedures for account mind action incorporate positron discharge tomography (PET), close infrared spectroscopy (NIRS), practical attractive reverberation imaging (fMRI),electrocorticography (ECoG), lectroencephalography (EEG), and magnetoencephalography (MEG). These strategies measure either metabolic (blood oxygenation) or electrophysiological associates of cortical action with various fleeting and spatial goals, and have various degrees of obtrusiveness. Subsequently, every technique has particular preferences and downsides [32]. fMRI: fMRI is a down to earth neuroimaging framework evaluating mind work by recognizing changes in circulatory system related with neural activity. The supposition that can't avoid being that neurons need more oxygen when they're dynamic. Since circulation system is moderate, fMRI is known to have low time objectives. In any case, the central nature of alluring resonation imaging is its extraordinary spatial objectives. Customarily, respondents need to lay unmoving in an appealing focus while a superconducting magnet is turning with high repeat around the body. fMRI by then gauges the modification in polarization between oxygen-rich and oxygen-poor blood, seeming relative activity of changed personality regions. Moreover, incredibly high-objectives pictures of cerebrum structures can be made with an excellent dimension of precision. With attractive imaging, you can reproduce the individual skull shapes and cortical layers of the majority of your respondents.

MEG: While EEG is recording the electrical development made by neural ending, MEG gets the alluring fields delivered by neural activity. MEG devices are absolutely stationary (like fMRI devices). They require the part to lay or sit for all intents and purposes unmoving and make light of advancements. MEG measures when in doubt need to occur in a shielded chamber to avoid appealing fields from the outside to intrude with the data recording. The best piece of elbowroom is that MEG combines the high precision in time of the EEG with the high precision in space of the fMRI. Finally, you get an accurate picture of the time objectives of the sign, which means you will know correctly which zones are dynamic and meanwhile you will know the structure of the skull and the cerebrum all around unequivocally.

EEG: When utilizing EEG, you are estimating electrical action created by the different cortical layers of the human cerebrum. In particular, electrical sign are gotten from dim issue areas, which have high densities of pyramidal cells that speak with one another. At whatever point enormous gatherings of these pyramidal cells are starting up in a synchronized example, the produced power emanates to the scalp surface this is the thing that we record with EEG cathodes. Since the electric fields are still extremely low, signals are by and large intensified. The action will be more grounded contrasted with a reference area (ordinarily situated behind the ears alluded to as the left/right "mastoid processes"). Keep at the top of the priority list that EEG is a referential chronicle and not a flat out voltage - it generally speaks to a relative increment or diminishing in power at the particular area.

\section{Published By:}


The adjustments in electric fields happen extremely quick so with EEG you will get bits of knowledge into mind forms with a high time goals (up to 1 millisecond reliant on the examining rate). EEG enables you to record cerebrum forms that happen soon after the beginning of visual or auditive improvements (there are steady mind forms officially after 50-100 ms post-upgrade), yet you can likewise screen cerebrum states reflecting commitment, inspiration or sleepiness over longer periods (hours to days). This magnificent time goals gives you bits of knowledge on the exact planning of mind handling.

PET: Portrayal PET tracers can likewise picture the movement of a specific class of synapses. An assortment of tracers, or ligands, exists, and more are consistently being created. The ligand is named with a radioisotope (generally $18 \mathrm{~F}$ and $11 \mathrm{C}$ ) and ties to a particular class of synapse receptor or synapse transporter. Synapse receptors get compound sign from different neurons with synapses tie to them; synapse transporters transport synapses starting with one neuron then onto the next over the neurotransmitter. This sort of imaging offers a non-obtrusive, non-terminal approach to consider the circulation and action of various synapse frameworks. Practically identical investigations in like manner neuroscience model species, for example, macaque monkeys and rodents, would utilize strategies that either require a well-saved after death cerebrum or include intrusive examinations, which makes this class of PET techniques a superior decision for people and extraordinary gorillas [36].

Reference channel: The vast majority of the accessible strategies need a committed ancient rarity channel to be practical. So as to evacuate ocularor cardiovascular ancient rarities, the reference channel frequently gives agreeable correlative data to distinguish ECG/EOG antiques. In addition, ongoing contact impedance estimation can give the correlative data about antiques because of anode pop, development or free association. Some development GPS beacons, for example, movement caught camera, accelerometer as well as gyrator can recognize movement ancient rarities.

EOG: By the use of EOG reference channel [44, 45, 46] we can remove EOG relics. In [46], a cross breed de-noising procedure has been represented which combines discrete wavelet change (DWT) and an adaptable marker filter (APF) for modified identification and departure of visual ancient pieces for helpful EEG applications. This gives the lower MSE and higher relationship among cleaned and one of a kind EEG in connection with existing methods, for instance, wavelet bundle change (WPT) and free portion examination (ICA), discrete wavelet change (DWT) and adaptable hullabaloo revocation (ANC).Another article [43] declared a robotized visual old irregularity clearing procedure using flexible filtering and ICA with the help of vertical (vEOG) and level (hEOG) EOG channel as reference. On the other hand, Flexer et al. [44] star spoke to an ICA-based visual old irregularity removal method from outwardly debilitated subjects' EEG utilizing both vertical and level EOG references.

ECG: Authors in [47] proposed clearing/lessening of ECG/heart ancient pieces from EEG using an alternate ECG reference channel. In [48], a customized procedure reliant on a modified ICA figuring has been suggested that works for a singular channel EEG and the ECG (as reference) which gives promising results when differentiated and two standard methodologies that use an imply ence channel to be explicit outfit typical subtraction (EAS)and flexible filtering. The other two articles proposed their methodologies for application in neonatal EEG checking. Another paper [49] proposed a blend of EMD and flexible filtering based methodology for ECG relic ejection in preterm EEG and reported up to $17 \%$ improvement in association coefficient among interesting and cleaned datasets differentiated and departure by simply adaptable filtering. Eye tracker: Both Kierkels et al. [50] and Noureddin et al. [51] reported techniques for ejection of visual collectibles by using an eye tracker as reference. The upside of using eye tracker is that it can lessen the undesired EEG winding conveyed by using an EOG channel as reference since EOG gets visual events just as some frontal EEG events. Moreover, in useful step by step applications, the use of eye tracker clears the essential of EOG terminals associated with the face. Results in [50] show significantly improved execution in removing of simply eye advancement collectibles by joining Kalman filter with the eye tracker information differentiated and three other surely understood methodologies specifically Regression, PCA, and SOBI. On the other hand, Noureddin et al. [51] introduced an online computation for visual doodads (the two advancements and flash) removal from EEG by utilizing a quick eye tracker $(>400 \mathrm{~Hz})$ close by the frontal EEG as reference as opposed to EOG channel. The article used two flexible filters (RLS and $\mathrm{H})$ to exhibit the efficacy of their proposed method, which was seemed to outmaneuver the techniques using only EOG as reference. Accelerometer: There are relatively few articles offered an explanation to have used accelerometer annals identified with EEG represents perceiving development ancient pieces [52, 53]. In [52], it has been shown that advancement ancient pieces can be perceived thus using an accelerometer with a made computation subject to AR showing and as such can grow the speed efficiency for customized estimation of EEG model parameters differentiated and manual disclosure of improvement relics. Sweeney uncovered in [53] that the usage of accelerometer as reference channel not solely can perceive development knick-knacks yet also can empty them with the use of different filtering methods, for instance, adaptable filters, Kalman filtering and Wiener filtering.

Gyrator: Authors in [54] proposed to recognize distinctive head development antiques naturally by utilizing a spinner as integral highlights in combination with EEG highlights and finally with the assistance of SVM, to group ancient rarities from neural data. The technique is enlivened by the acknowledgment of an ancient rarity discovery framework for executing with the purpose of consideration REACT (Real-time EEG Analysis for occasion identification) innovation that has potential application in the recognition of neurological occasions (for example seizure occasions) in grown-ups. The ancient rarities were produced for 10 unique kinds of head related developments utilizing 14-channel Emotiv EEG headset and the development time was recorded for approval during relic location. 
The revealed exactness regarding Avg. ROC territories was 0.802 and 0.907 for member autonomous and subordinate frameworks separately.

Contact impedance measurement: Bertrand et al. furthermore, Mihajlovic et al. [55,56] announced that by estimating the adjustment in contact impedance because of head developments can evaluate the movement relics and by using this data with a versatile filter in blend with band-pass filtering, the curios can be diminished significantly continuously. The article likewise contemplates the impact of head development ancient rarities on EEG accounts brings about sullying the phantom area in $<20 \mathrm{~Hz}$ recurrence.

Motion captured camera: Authors in [57] proposed a channel and IC-based strategy to expel development ancient rarities during strolling and running from a high-thickness EEG chronicles (248-channel) with the assistance of kinematics and energy data procured from a8-camera, 120 edges/s, movement catch framework. The subject was approached to walk and keep running on a custom fabricated, double belt power estimating treadmill with two 24 in wide belts mounted flush with the floor while at the same time both cerebrum and body elements were recorded. The findings infer that high-thickness EEG is conceivable to use so as to study mind elements during entire body developments; and the curio from musical step occasions can be diminished by format relapse methodology.

Robustness: Power is a significant issue in building up any antiquity expulsion calculation as curios are of assorted kinds and sully the EEG contrastingly in various chronicle situations. A portion of the elements that ought to be considered for strength incorporate curio SNR, sort of antique, span of curios, subject-changeability, ecological inconstancy, application-specificity.

\section{CONTROL SIGNALS TYPES IN BCI}

Various examinations have portrayed a huge gathering of cerebrum flag that may fill in as control flag in BCI frameworks. In any case, just those control sign utilized in current BCI frameworks is talked about underneath: moderate cortical possibilities, P300 evoked possibilities, visual evoked possibilities and sensorimotor rhythms. All the sign controls are recorded in Table 5.1 alongside a portion of their fundamental highlights.

TABLE 3 SUMMARIES OF CONTROL SIGNALS

\begin{tabular}{|l|l|l|l|l|}
\hline Signal & \multicolumn{1}{|c|}{ Physiological phenomena } & \multicolumn{1}{|c|}{$\begin{array}{l}\text { Information } \\
\text { transfer rate }\end{array}$} & $\begin{array}{c}\text { Number of } \\
\text { choices }\end{array}$ & \multicolumn{1}{|c|}{ Training } \\
\hline SCP & $\begin{array}{l}\text { Slow voltages shift in the brain } \\
\text { signals }\end{array}$ & $5-12$ bits/min & $\begin{array}{l}\text { Low (2 or 4, } \\
\text { very } \\
\text { difficult })\end{array}$ & Yes \\
\hline P300 & $\begin{array}{l}\text { Positive peaks due to infrequent } \\
\text { stimulus }\end{array}$ & $\begin{array}{l}20-25 \\
\text { bits/min }\end{array}$ & High & No \\
\hline VEP & $\begin{array}{l}\text { Brain signal modulations in the } \\
\text { visual cortex }\end{array}$ & $\begin{array}{l}60-100 \\
\text { bits/min }\end{array}$ & High & No \\
\hline $\begin{array}{l}\text { Sensorimotor } \\
\text { rhythms }\end{array}$ & $\begin{array}{l}\text { Modulations in sensorimotor } \\
\text { rhythms } \\
\text { synchronized to motor activities }\end{array}$ & $3-35$ bits/min & Low (2, 3, 4, 5) & Yes \\
\hline
\end{tabular}

Slow Cortical Potentials (SCPs): Utilizing an idea interpretation gadget individuals can be prepared to produce intentional SCP changes [6].The thought-interpretation gadget is an apparatus utilized for self-guideline SCP preparing, so this shows visual-sound-related stamps with the goal that the client can figure out how to move the SCP. The idea interpretation gadget normally contains a cursor on a screen so that the vertical position of the cursor always mirrors the sufficiency of SCP shifts. Albeit most idea interpretation gadgets show nonstop input. Likewise without persistent input it is conceivable to prepare SCP self-regulation [6]. Accomplishment in SCP self-guideline preparing relies upon various variables, for example, the patient's physical state and mental, social setting, inspiration, or the coach persistent relationship. It is realized that the learning ability of the client radically influences SCP regulation preparing. Self-guideline preparing is along these lines firmly prescribed for patients at the beginning period of a dynamic sickness. Commonplace precision rates accomplished for SCP grouping are worthy and it fluctuate between $70 \%$ and $80 \%$, however SCP-based BCI are generally gives the rates of data low. Plus, longer preparing is

required to utilize SCP-based BCI and all things considered, clients will require constant practice for a while [6].

P300 Evoked Potentials: Because of inconsistent sound-related, visual, or somatosensory upgrades in the EEG signal P300 evoked possibilities are available in positive pinnacles. Hardly any examination demonstrated that the less likely the upgrade, the bigger the adequacy of the reaction crest. Be that as it may, the exhibition might be diminished on the grounds that the when client becomes accustomed to the rare improvement and subsequently P300 adequacy is diminished .One focal points utilizing P300 based BCI is does not require preparing. $\mathrm{P} 300$-based $\mathrm{BCI}$ give an exceptionally low rate of data transmission in light of the fact that the classifier dependent on a normal is excessively basic, and the precision of P300 potential discovery is excessively low. Data transmission rates given by $\mathrm{P} 300$-based BCI can be likewise improved by thinking about the $\mathrm{BCI}$ as a boisterous transmission framework. BCI can in this way profit by the utilization of blunder redressing codes. 
Execution of the visual P300-put together BCIs depends not just with respect to the P300-evoked potential, yet in addition on the VEP reaction that, thus, unequivocally relies upon eye-stare heading [6].

Table 4 Features of VEP modulations: t-VEP, f-VEP and, c-VEP

\begin{tabular}{|c|c|}
\hline VEP modulation & Features \\
\hline t-VEP & $\begin{array}{l}\text { - } \text { Relatively low information } \\
\text { transfer rate }(<30 \text { bits/min) } \\
\text { - } \quad \text { No user training required } \\
\text { - Synchronous signal is necessary }\end{array}$ \\
\hline f-VEP & $\begin{array}{l}\text { - High information transfer rate } \\
\text { (30-60 bits/min) } \\
\text { - No user training required } \\
\text { - } \text { Simple system configuration } \\
\text { - More suitable for application } \\
\text { with few options }\end{array}$ \\
\hline c-VEP & $\begin{array}{l}\text { - } \text { Very high information transfer } \\
\text { rate ( }>100 \text { bits/min) } \\
\text { - User training required } \\
\text { - Synchronous signal is necessary } \\
\text { - More suitable for application } \\
\text { with many options }\end{array}$ \\
\hline
\end{tabular}

Visual Evoked Potentials (VEPs): VEPs are mind development adjusts that occur by tolerating a visual lift in the visual cortex. These changes are decently easy to perceive since the adequacy of VEPs augments tremendously as the update is moved closer to the central visual field. VEPs may be assembled by three novel criteria: (1) by the morphology of the optical overhauls, (2) by the repeat of visual prompting; and (3) by field instigation. As demonstrated by the essential guideline, using streak prompting or using reasonable models, for instance, entryway, checkerboard cross segment, and sporadic spot guide cause the VEPs. According to the repeat, VEPs can similarly be appointed transient VEPs (TVEPs) and as tireless state VEPs (SSVEPs). TVEPs happen when the repeat of visual actuation is underneath $6 \mathrm{~Hz}$, while SSVEPs occur in light of increases in a higher repeat. As shown by the third measure, dependent upon the zone of on-screen support VEPs can be divided into whole field VEPs, half field VEPs, and part field VEPs [6]. Again depending upon the specific lift progression equalization being utilized [30] SSVEP-based BCIs can be organized into three orders those are time changed VEP (t-VEP) BCIs, repeat balanced VEP (f-VEP) BCIs, and pseudorandom code managed VEP (c-VEP) BCIs. The blast plans for different targets are either cautiously non-covering or stochastic in a t-VEP BCI that infers flash groupings of different targets are symmetrical in time. In a $\mathrm{f}-\mathrm{VEP} \mathrm{BCI}$, every goal is flashed at a novel repeat by creating an irregular gathering of evoked responses with a comparable focal repeat as its music. Pseudo-subjective progressions are used in a c-VEP BCI. The range of ON and OFF states of every target's flicker is directed by a pseudorandom gathering. Table 5.3.1 exhibits the diagram of each alteration.

Sensorimotor Rhythms (mu and beta rhythms): Sensorimotor rhythms contain mu and beta rhythms, which are movements in the mind activity limited in the mu band its repeat range is $7-13 \mathrm{~Hz}$, generally called the Rolandic band, and beta band repeat range is $13-30 \mathrm{~Hz}$, independently. The two rhythms are connected with the goal that some beta rhythms are symphonious mu rhythms, though some beta rhythms may moreover be self-ruling. The adequacy of the sensorimotor rhythms varies when cerebral activity is related to any motor assignment but certifiable improvement isn't required to adjust the ampleness of sensorimotor rhythms. Near parity structures in the motor rhythms are conveyed in view of mental routine with regards to a motor exhibition with no obvious motor yield [6]. Sensorimotor rhythms have been used to control BCIs, in light of the way that people can make sense of how to deliver these alterations purposely in the sensorimotor rhythms [6]. Sensorimotor rhythms can drive forward through two sorts of abundancy alterations known as event related desynchronization (ERD) and event related synchronization (ERS) that are created substantial induction, motor direct, and mental imagery [36]. ERD incorporates a plentifulness covering of the rhythm and ERS construes abundancy update.

Discovery of ERD is utilized to distinguish when a BCI member is endeavoring engine symbolism (MI) and in this way shapes the MI-BCI control. ERS happens while the mu beat is as yet weakened. This is another musicality identified with engine errands also [6]. Sensorimotor rhythms have been explored irrelevantly in BCI inquire about. Understood BCI frameworks, for example, Wadsworth, Berlin, or Graz. BCIs utilize sensorimotor rhythms as control signals. The BCIs dependent on sensorimotor rhythms can work in either synchronous or offbeat mode. The most recent relapse in the field of BCIs dependent on sensorimotor rhythms have demonstrated that it is conceivable to anticipate human intentional developments before they happen dependent on the adjustments in sensorimotor rhythms. Moreover, this forecast could be given without the client making any developments whatsoever.

Electroencephalography (EEG):

An electroencephalogram is a test used to assess the electrical movement in the cerebrum. Synapses speak with one another through electrical driving forces. The Human mind is the most unpredictable piece of the entire body. It produces diverse sort of mind waves in various mental states. These waves help in better comprehension of the human exercises, for instance hand or leg development, eye flicker and so on. Cerebrum waves have entranced numerous analysts. There has been consistent improvement in the advancement of human machine collaboration framework with the assistance of EEG signals. Ordinary EEG show up in grown-ups who are wakeful, the EEG indicates commonly alpha waves and beta waves. The different sides of the mind show comparable examples of electrical movement. There are no irregular blasts of electrical action and no moderate cerebrum waves on the EEG following. On the off chance that blazing lights (photic incitement) are utilized during the test, one region of the mind (the occipital locale) have a concise reaction after each glimmer of light, however the cerebrum waves are ordinary. EEG is said to be unusual when various examples of electrical action are acquired from the different sides of cerebrum. This speak to an issue in one region or side of the mind is available. The EEG demonstrates unexpected blasts of electrical action (spikes).

Published By: 
These abrupt changes might be brought about by a cerebrum tumor, damage, epilepsy. At the point when an individual has epilepsy, the area and accurate example of the irregular mind waves may help show what sort of epilepsy or seizures the individual has. In numerous individuals with epilepsy, the EEG may show up totally ordinary between seizures. The EEG records changes in the cerebrum waves that may not be in only one zone of the mind. Employments of EEG is as pursue

> Diagnose epilepsy and see what kinds of seizures are happening. EEG is the most valuable and significant test in affirming a finding of epilepsy. - Check for issues with loss of awareness or dementia.

$>$ Help discover an individual's possibility of recuperation after an adjustment in cognizance.

$>$ Find out if an individual who is in a trance like state is cerebrum dead.

$>$ Study rest issue, for example, narcolepsy.

$>$ Watch cerebrum movement while an individual is accepting general anesthesia during mind medical procedure. like issues in the cerebrum, spinal line, or sensory system.

$>$ Points of interest of EEG is given beneath

$>$ There are different bit of leeway of EEG flag some of them can be expressed as pursues:

$>$ Temporal goals of EEG sign is high.

$>$ EEG measures the electrical action legitimately.

$>$ EEG is a non-obtrusive methodology.

$>$ EEG has capacity to investigate cerebrum movement.

$>$ Major weakness is EEG that it is hard to discover the wellspring of electrical movement from where it is turning out.

Detection of an EEG signals: Neurons in our cerebrum begins correspondence through an electrical driving forces. So as to gain the cerebrum waves the quantity of cathodes are set on the scalp area to quantify the abundancy of electric motivation. Recurrence scope of a typical EEG sign is 1 $\mathrm{Hz}-100 \mathrm{~Hz}$ however the $100 \mathrm{~Hz}$ is uncommon and adequacy goes between $10 \mu \mathrm{V}-100 \mu \mathrm{V}$. For the most part, the sign experiences poor spatial goals and low sign to-commotion proportion (SNR) of any evoked reaction which gets implanted inside on-going foundation action [21]. At the point when EEG sign is examined has an exceptionally low recurrence run in hertz. EEG sign changes as indicated by the cerebrum action states. Contingent upon these states, we can recognize a few rhythms (waves) in view of the recurrence groups. The various rhythms of mind are gamma, beta, alpha, theta and delta [21]. These rhythms alongside their abundancy, recurrence reaches and particular perspective are given in Table 5 . The wave shapes for these rhythms are appeared in Figure.1.

Sources of Noise in EEG Signal: Fig 3 outlines the wellsprings of in EEG signals. One of the difficulties in utilizing EEG is the extremely little sign to-clamor proportion (SNR) of the mind flag that we are attempting to watch, any sign other than that of intrigue could be named as a curio or just commotion. EEG sign experiences principally two sorts of clamor. The main sort, physiological ancient rarities which emerge from an assortment of body exercises that are either because of solid developments, other skin obstruction
$>$ Help see whether an individual has a physical issue

variances or bioelectrical possibilities. The transcendent physiological curios incorporate electrooculargraphic movement (EOG, eye), scalp recorded electromyographic action (EMG, muscle), electrocardio-realistic action (ECG, heart), ballistocardiographic action (heart-related pulsatile movement) and breath. These ancient rarities are constantly present somewhat and are regularly significantly more unmistakable on the scalp than the naturally visible cerebral possibilities. This outcomes in an unfortunate negative sign to-clamor proportion in the EEG. Second kind of commotions incorporates outside, natural wellsprings of clamor, for example, AC electrical cables, lighting and a huge cluster of electronic gear from PCs, presentations and TVs to remote switches, scratch pad and cell phones. The most essential strides in managing ecological commotion are evacuating any pointless wellsprings of electro-attractive (EM) clamor from the account room and supplanting gear utilizing substitute current with hardware utilizing direct current, for example, direct current lighting. A further developed and exorbitant measure is to protect the account room from EM clamor by utilization of a Faraday confine [22, 23].

Data Collection through Electrodes: Brain activity is obtained by different types of electrodes. This elctrodes are placed on the scalp region. Fig 4.3.1 illustrates the placement of electrodes which commonly follows the 10-20 system and it may be extended to 32, 64, 128, or 256 placement of electrodes [24]. During the last 3 years, to maximize the performance of such electrodes some research work has been done. In a recent work of Mihajlovic and Peuscher J [25], they demonstrated that dry and water-based electrodes can replace gel ones in BCI applications where it lowers a communication speed which is acceptable. Hazrati and H.M., Hofmann [8], they presented a wireless EEG monitoring system. The system is capable of processing brain signals on-board recorded from non-contact sensors. It provides an excellent option for developing a compact BCI with a direct connection to the external device e.g. robot. The problem of interference pick-up by electrodes was addressed by the recent work of [27].

\section{VARIOUS APPROACHES FOR REMOVAL OF ARTIFACTS}

Blind source separation (BSS)

Blind source separation or BSS is a way to estimate different components from the mixtures of these signals. This method is called 'blind' because of the absence of any information except mixture. There are several techniques in blind source separation. The idea behind BSS analysis is to

\begin{tabular}{|l|l|l|l|}
\hline $\begin{array}{l}\text { Rhythm } \\
\text { Waves }\end{array}$ & Amplitude & $\begin{array}{l}\text { Frequenc } \\
\text { y range }\end{array}$ & State of mind \\
\hline Gamma & Less than 5 & $\begin{array}{l}\text { Above } \\
31 \mathrm{~Hz}\end{array}$ & $\begin{array}{l}\text { Mechanism of } \\
\text { consciousness }\end{array}$ \\
\hline Beta & $30-$ May & $13-30 \mathrm{HZ}$ & $\begin{array}{l}\text { Active thinking, active } \\
\text { attention, alert }\end{array}$ \\
\hline Alpha & $30-50$ & $8-13 \mathrm{~Hz}$ & Relaxed awareness \\
\hline Theta & $\begin{array}{l}\text { More than } \\
20\end{array}$ & $4-8 \mathrm{~Hz}$ & $\begin{array}{l}\text { Emotional stress, } \\
\text { drowsiness and sleep in } \\
\text { adults }\end{array}$ \\
\hline Delta & $\begin{array}{l}\text { High } \\
\text { amplitude } \\
(20-200)\end{array}$ & $0.5-4 \mathrm{~Hz}$ & Deep sleep \\
\cline { 3 - 4 } & & & \\
\hline
\end{tabular}


produce components that correspond to distinct neural and nonneural activity [37, 38, 39, 40].

Principle Component Analysis (PCA): Main part examination or PCA is a strategy that utilizations measurable strategies oforthogonal change which is utilized to change the watched segment of uncorrelated variable to segments of uncorrelated factors. These factors are known as called essential parts. These central segments are not exactly the first factors. The central segments have the difference in diminishing request. The segment watched first has most elevated change pursued by second, etc. Additionally, there ought to be symmetry between these factors. PCA is a kind of spatial filter that changes the time area datasets into an alternate space by pivoting tomahawks in a N-dimensional space (where $n$ is the quantity of factors or EEG channels) with the end goal that each measurement in the new space has least fluctuation and the tomahawks are symmetrical to one another [17]. PCA decreases information measurement and features specific highlights of information, which is typically difficult to recognize in the spatially unfiltered information as the new segments are made by weighted blends of all EEG channels. Two ongoing papers proposed antiquity evacuation technique dependent on PCA: Turnip [42] detailed the utilization of hearty PCA after preprocessing is done base wavelet de-noising and band- pass-filtering; while Turnip and Junaidi [43] contrasted PCA and ICA for ancient rarity expulsion and discovered ICA performs superior to PCA.

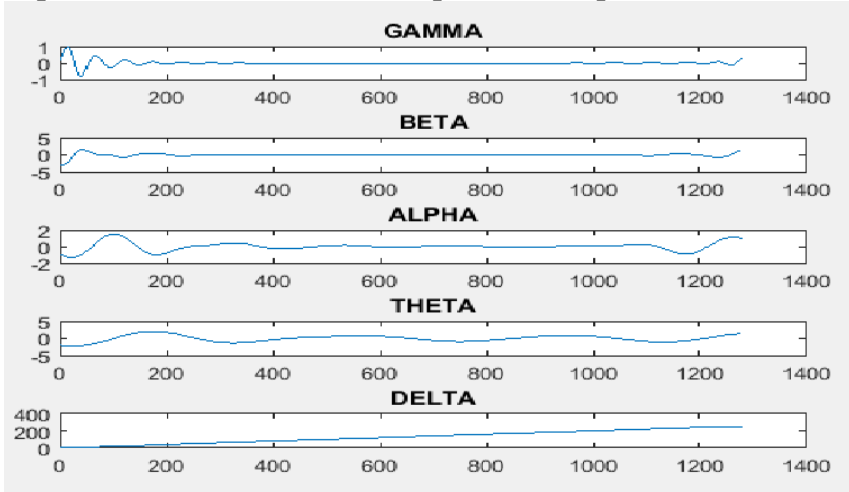

Fig 2 Different types of brain waves

(a)
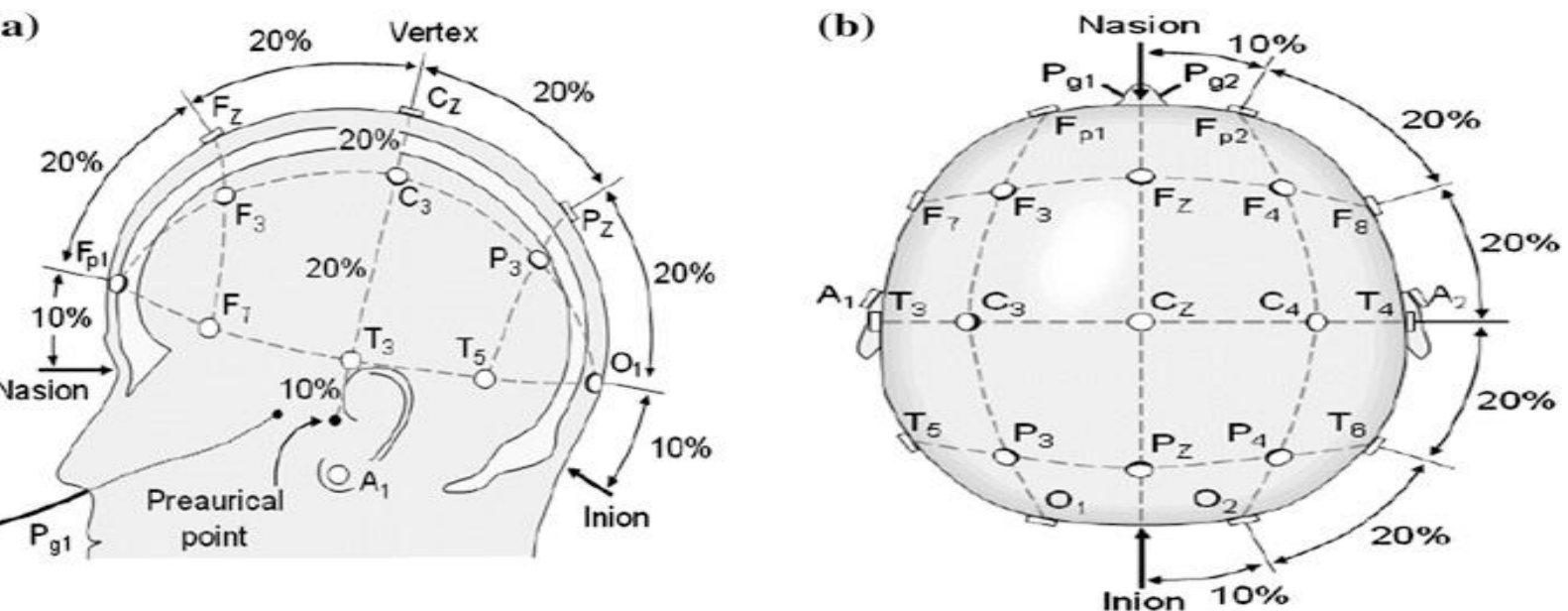

Fig 4 Electrode placement over scalp region followed by 10/20 system.

a) Left side view b) Top view[6] 


\section{Automated Artifact Removal in EEG Signals of Brain Computer Interface using Wavelets and ICA}

\section{Independent Component Analysis (ICA):}

Independent Component Analysis or ICA is a system to detach the mix of various sign into different included substance free part. These parts are accepted of non-Gaussian and self-sufficient in nature. It wears down the higher degree work. This is the impelled methodology of Principal Component examination. Allow us to consider a sign is gotten with the mix of different sign. This sign is addressed as $\mathrm{Y}=\mathrm{A} \_$sy=A_s Here $\mathrm{Y}$ is the gotten sign, is the source banner and is the different parameters depend on different components. Since getting the principal source signal from the mix we have to figure the opposite of the lattice. This opposite of is addressed as W. $\mathrm{s}=\mathrm{WY}$ We have to figure this $\mathrm{W}$ with the help of different estimation. A bit of the estimations SCICA, AWICA, ORICA, JADE-ICA, jdICA, SOBI, fastICA, etc are used.

Wavelet Transform: Wavelet changes has a bit of leeway over Fourier changes is that it catches both recurrence and time data which is restricted to recurrence data in the Fourier change. This technique likewise has advantage over BSS, EMD and EEMD. This strategy has quicker execution time when contrasted with different techniques. Wavelet change can be communicated as

$$
\begin{array}{ll}
F(a, b)= & \int_{\infty}^{\infty} f(x) \psi_{a, b}(x) d x \\
F(a, b)=\int_{\infty}^{\infty} f(x) \psi_{a, b}(x) d x &
\end{array}
$$

\section{Discrete Wavelet Transform :}

Discrete Wavelets Transform (DWT) is the usage of wavelet change over discrete arrangements of wavelet scales. Wavelets in DWT are commonly symmetrical to one another. The scaling capacity is characterized as

$\phi(x)=\sum_{k=-\infty}^{\infty} a_{k} \phi\left(S_{x}-k\right)$

Here $\mathrm{S}$ is scaling factor and must be greater than 2. And orthogonality in the DWT can be defined as

$$
\begin{aligned}
& \int_{\infty}^{\infty} \phi(x) \phi(x+1) d x \\
& \delta_{0.1} \int_{\infty}^{\infty} \phi(x) \phi(x+1) d x=\delta_{0.1}
\end{aligned}
$$

\section{Stationary Wavelet Transform}

Stationary Wavelet Transform or un destroyed wavelet change is a strategy of interpretation - invariance adjustment. Annihilation of coefficient each degree of change is done in SWT. In old style DWT, the interpreted sign isn't equivalent to contrasted with DWT of the first sign. Along these lines, traditional DWT isn't move invariance. Thus, this issue of move invariance is finished by expelling the down-samplers and up-samplers in the DWT. Likewise up-examining of the coefficients by a factor of the in jth level. In SWT the quantity of yield and number of information sources are equivalent. This makes this plan repetitive. It is important for $\mathrm{N}$ level disintegration there should same excess so as to identify the change [35]. A basic methodology for antiquities expulsion is high pass separating the information to expel EOG and low pass sifting the information to evacuate [5].This technique will expel any mind sign of enthusiasm for a similar

recurrence extend. On the off chance that a solid reference channel is accessible, for instance, an EOG recording, relapse in the time, or recurrence area can be performed. The disservice of this strategy is that EOG accounts frequently contain cerebrum signals which would definitely likewise be expelled. In any case, it has been demonstrated this is best over relic dismissal techniques in light of the decreased measure of information misfortune [28, 29]. In principle, an assortment of techniques may be utilized to decrease or wipe out EMG tainting. These incorporate generally basic strategies, for example, dismissal of EEG portions that surpass a predefine edge sufficiency (Brunner et al., 1996; Anderer et al.,1999; Junghofer et al., 2000) or direct non-straight low-pass separating (Barlow, 1986; Ives and Schomer, 1988; Panych et al., 1989; Sadasivan and Dutt, 1995; Klass, 1995) and increasingly modern techniques, for example, factor deterioration utilizing essential part (Lagerlund et al., 1997) or free segment investigation (Jung et al., 2000). These referenced techniques requires definite learning of the phantom and land examples of cranial EMG tainting. This data isn't accessible at present. Henceforth these techniques are very little usable. An elective methodology is to utilize visually impaired source partition (BSS), which depends on assessing the basic sources (segments) that make the sign estimated with the EEG. Preferably, the evaluated parts contain either curios or EEG movement. It is then conceivable to expel antiquities by end of the relating parts. Makeig was one of the first to show the likelihood of applying ICA strategies to perform BSS on EEG information [5]. An impediment characteristic inside the present investigation is that it utilizes a recreation that, similar to all reenactments, is more straightforward than this present reality and may not sum up well to genuine information. Ancient rarities were effectively isolated into a couple of yield segments and expelled from the information. Points of interest of utilizing ICA to expel EOG ancient rarities from EEG information, rather than dismissal or relapse based strategies, have been appeared a few examinations, demonstrating that the level of cerebrum signal that is evacuated with the EOG is diminished. EOG ancient rarity evacuation based on disengagement into autonomous parts (ICs) utilizing BSS has been shown in [5]. So in this work the exhibition of the most encouraging techniques for robotized amendment and expulsion of relics from the EEG are explored and thought about. The Lagged Auto-Mutual Information Clustering (LAMIC), depends on the closeness of the transient elements of the sign as portrayed by their auto-common data. Wavelet change utilizes wavelet disintegration and remedy of the sufficiency of the detail coefficients relating to antiquities and MSSA performs well at low SNRs however to the detriment of an enormous number of false positive amendments.

\section{LITERATURE SURVEY}

Fabien Lotte and Cuntai Guan [13] have depicted the Learning spatial channels that expand the change of band pass sifted EEG signals. 
Hyohyeong Kang, and Yunjun Nam [12] have given ideas of improve arrangement execution over the standard CSP, particularly for the situation less number of preparing tests. $\mathrm{H}$. Nolan, R. Whelan, R.B. Reilly [7] presented the Fully Automated Statistical Thresholding for EEG ancient rarity Rejection (FASTER) which consolidates Independent Component Analysis (ICA) technique. Quicker technique is tried crosswise over various quantities of scalp terminals (128, 64 and 32). The consequences of the investigation of the recreated information spoke to that the FASTER had commonly high affectability and particularity for discovery of antiques. S. P. Fitzgibbon [20] utilized a visually impaired sign partition (BSS) calculations for isolating basic kinds of tainting from EEG. This BSS seems, by all accounts, to be best at isolating muscle and squint sullying. So BSS calculation is increasingly viable and useful asset for isolating and expelling sullying from EEG. Its nature of the division is exceedingly subject to the sort, level of defilement and the decision of BSS calculation. Xinyi Yong, Mehrdad Fatourechi [19] have proposed an antiquity recognition calculation to distinguish various sorts of curios in a self-managed BCI framework. This calculation is fundamentally founded on a straightforward the sufficiency of EEG signals and thresholding strategy. Sebastian Halder, Michael Bensch and Wolfgang Rosenstiel presented the antiquities expulsion technique dependent on Support Vector Machine (SVM). This technique is prepared to arrange EMG and EOG ancient rarities, which empowers the use of the strategy as a channel in estimations with online criticism [5]. M. K. Islam et al. chipped away at various calculations to naturally identify and dispense with the ancient rarity from the crude EEG. [38] In this strategy SWT is utilized for the end of antiquity. This strategy is additionally contrasted and the ICA, wICA, wCCA, EMD-ICA and EMD-CCA. Results are better on account of SWT. [39]In this technique WT is utilized for the disposal of antiquity. T. Radüntz, J. Scoutena, O. Hochmuth and B. Meffert presented another mechanized methodology ancient rarity end utilizing straight discriminant examination (LDA) for arrangement of highlight vectors separated from ICA segments by means of picture preparing calculations. This technique got $88 \%$ of precision. Contrasted with the current robotized arrangements this calculation has two fundamental points of interest that is it isn't constrained to a particular number or sort of curio. What's more, different focal points over other technique is that it doesn't rely upon direct chronicle of ancient rarity signals [21]. Table 6 demonstrates the writing study dependent on the different strategies they proposed and calculations displayed by different writers together with their fundamental reason and their outcomes.

\section{A Review On Preprocessing Techniques That Deals With The Artifacts Of EEG Signals:}

A BCI is a man-made brainpower framework that can perceive a specific arrangement of examples in cerebrum sign after successive stages: signal obtaining, preprocessing or signal upgrade, highlight extraction, characterization [1]. So as to perform include extraction and arrangement from mind waves, it is basic to pre-process the crude cerebrum waves for expanding the sign to clamor proportion and improve the pertinent data dug in the EEG signal. Positively, electrical movement of the eyes or of the muscles or high recurrence clamor from electrical net ( $50 \mathrm{~Hz}$ in Sweden) influences the EEG sign and makes them uproarious. The electrical movement of the muscle or eyes causes more unsettling influences as they have enormous sufficiency when contrasted with EEG signal. One must be cautious while

TABLE 8.1: Literature Survey

\begin{tabular}{|c|c|c|c|c|}
\hline Ref no. & Author name & Method & Purpose & Result \\
\hline [04] & $\begin{array}{l}\text { Ian Daly and M. } \\
\text { Billinger }\end{array}$ & $\begin{array}{l}\text { Based upon sensorimotor rhythm } \\
\text { modulation \& steady state visual } \\
\text { evoked potential. }\end{array}$ & $\begin{array}{l}\text { To assist the communication needs of } \\
\text { cerebral palsy patients. }\end{array}$ & $\begin{array}{l}\text { Providing better BCIs method to control } \\
\text { needs of individuals with CP. }\end{array}$ \\
\hline [05] & $\begin{array}{l}\text { Sebastian } \\
\text { Halder }\end{array}$ & BSS/ICA method & Classify EMG and EOG artifacts. & $\begin{array}{l}\text { Using filters that removes the artifacts } \\
\text { from BCI data. }\end{array}$ \\
\hline [07] & H. Nolan & Unsupervised method & EEG artifact detection \& rejection. & $\begin{array}{l}\text { Quantify the utility and performance of } \\
\text { FASTER. }\end{array}$ \\
\hline [08] & $\begin{array}{l}\text { Ian Daly and } \\
\text { Nicolaou }\end{array}$ & Wavelets BSS and MSSA & $\begin{array}{l}\text { To removing blink, ECG and EMG } \\
\text { artifacts from the EEG. }\end{array}$ & $\begin{array}{l}\text { Three methods are introduced for } \\
\text { automated artifact correction in the EEG. }\end{array}$ \\
\hline [09] & $\begin{array}{l}\text { P. Senthil } \\
\text { Kumar }\end{array}$ & Statistical method & $\begin{array}{l}\text { To Identify the spikes and Ocular } \\
\text { artifact spikes zones. }\end{array}$ & $\begin{array}{l}\text { Artifacts of EEG are removed with the } \\
\text { help of wavelet decomposition. }\end{array}$ \\
\hline$[10]$ & $\begin{array}{l}\text { Veer Amol } \\
\text { Motinath }\end{array}$ & Electrocardiogram data Compression & $\begin{array}{l}\text { Mother wavelet selection based on } \\
\text { minimum percentage root mean } \\
\text { square difference. }\end{array}$ & $\begin{array}{l}\text { Good compression performance in ECG } \\
\text { signals in terms } \\
\text { PRD,SNR,QS,PRDN\& RMS. }\end{array}$ \\
\hline [14] & Eugene Teo & \multirow[t]{2}{*}{ P300 classification methods } & \multirow{2}{*}{$\begin{array}{l}\text { Media Communication } \\
\text { Center for the paralyzed } \\
\text { people. }\end{array}$} & \multirow{2}{*}{$\begin{array}{l}\text { Improve the overall } \\
\text { reliability of BCI systems. }\end{array}$} \\
\hline & & & & \\
\hline [15] & $\begin{array}{l}\text { Akaysha } \quad \text { C. } \\
\text { Tang }\end{array}$ & $\begin{array}{l}\begin{array}{l}\text { Second-order blind identification } \\
\text { (SOBI) }\end{array} \\
\end{array}$ & $\begin{array}{l}\text { To decompose mixtures of signals into } \\
\text { a set of components. }\end{array}$ & $\begin{array}{l}\text { Improves the SNR and reduce the level of } \\
\text { subjectivity }\end{array}$ \\
\hline$[16]$ & I. Goncharova & Independent component analysis(ICA) & $\begin{array}{l}\text { To describe both average data and the } \\
\text { range of individual differences }\end{array}$ & $\begin{array}{l}\text { Detecting and eliminating EMG } \\
\text { Contamination. }\end{array}$ \\
\hline [17] & Joseph T & Innovative methodology & $\begin{array}{l}\text { To study brain dynamics during whole } \\
\text { body movements. }\end{array}$ & $\begin{array}{l}\text { Mechanical artifact from rhythmic gait } \\
\text { events minimized using template } \\
\text { regression procedure. }\end{array}$ \\
\hline [18] & $\begin{array}{l}\text { Hossein } \\
\text { Mamaghanian }\end{array}$ & acquisition/compression & $\begin{array}{l}\text { Low-complexity energy-efficient ECG } \\
\text { compression on the state-of the art } \\
\text { Shimmer WBSN mote. }\end{array}$ & $\begin{array}{l}\text { EEG signals are compressed using DWT } \\
\text { based counterpart for good reconstruction } \\
\text { quality. }\end{array}$ \\
\hline
\end{tabular}


expelling these clamors from EEG signals; inadvertently it might happen to evacuate the important data settled in.

Similarly, the momentous part is evacuating the sign stimulated because of foundation mind action unessential to improve the parameters like sign to commotion proportion (SNR), Peak Signal to Noise Ratio (PSNR), and Mean Square Error (MSE). Also, this pre-handling helps in improving the presentation of the framework by isolating the clamors like Gaussian commotion, poisson commotion, white noise,.etc from the real sign. the sign of our advantage. Pre-handling is significant strides in EEG sign preparing. Pre-preparing systems help to expel.

\section{Mean Square Error:}

The MSE among the unique image $\mathrm{f}(\mathrm{x}, \mathrm{y})$ and the reassembled image $\mathrm{g}(\mathrm{x}, \mathrm{y})$ is given by

$M S E=\frac{1}{X X Y} \sum_{x=0}^{X-1} \sum_{y=0}^{Y-1}\left(f(x, y)-g(x, y)^{2}\right.$

1

Here XXY signifies the size of the image.

The image MSE is an quantity of great utility as to provides an average value of the energy last in the process of the original image in a situation where a person who observes two images that have goes through the same degradation is prove to fudge the one with the smaller MSE as closer to the original image signal it is possible for a very small MSE to be taken for the image as very close to the original signal. However a fact that cannot be ignored or last sight of is the presence of some problems when a comparison is made between images with degradation of various types.

Signal to Noise Ratio (SNR):

SNR is another measure that finds extending use in the comparison of the performance of reproduced images. It is defined as the power ratio between a signal (image) and the back ground noise.

$S N R=\frac{P_{\text {signal }}}{P_{\text {roise }}}$

$\mathrm{P}=$ average power.

Signal and noise power must be measured at the same points in a system.

$S N R=\frac{P_{\text {signal }}}{P_{\text {noise }}}=\left(\frac{A_{\text {signal }}}{A_{\text {noise }}}\right)^{2}$

3

Where A = RMS value of signal.

SNR is expressed using logarithmic decibel scale, therefore $S N R_{d b}=10 \log _{10} \frac{P_{\text {signal }}}{P_{\text {noise }}}=P_{\text {signal }}$
$P_{\text {moige }}(d B)$ And $S N R=\frac{\mu}{\sigma}$

Where, $\mu=$ signal mean or expected value and $\sigma=$ standard deviation of noise. SNR is also referred to as the sensitivity of the imaging system control of noise is possible through reducing it. The enhancement is done by averaging measurements. Measurement of SNR is done in $\mathrm{dB}$, which is a good indication of SNR reproduction the peak to signal ratio is another significant sub objective quantitative measurement. High value of quality score indicates high compression performance with less error [18].

Peak Signal to Noise Ratio (PSNR): Another significant parameter is the pinnacle sign to clamor proportion, which is the proportion of the greatest conceivable intensity of a sign to undesirable antiquities from the EEG sign and henceforth

the intensity of the commotion that adulterates influencing the dependability of its portrayal. PSNR discovers broad use as the measuring stick for the proportion of the nature of picture remaking. The first information is viewed as the picture while clamor is the portrayal of the blunder presented by the procedure. The portrayal of PSNR is as a general practices done in logarithmic decibel scale. A run of the mill estimation of PSNR is a lossy picture that is between $30-50 \mathrm{db}$. MSE is zero when two pictures are discovered indistinguishable. PSNR is communicated as far as db. PSNR is known for its measure that has prevalence. Other measure like SNR thinking of it as utilizations a steady an incentive for making correlation of the commotion rather than a sign referred to for variance as observed in SNR. This empowers important treatment. Treatment to PSNR values when quantitative correlation of various pictures is finished. Factual estimations for the separating systems are finished by following formulae. In table 5 Where $f(i, j)$ is original image. $F(i, j)$ is the enhanced image $\sigma^{2}$ is the variance of original image and $\sigma_{e}^{2}$ is the variance of enhanced image. The noise level is measured by the standard deviation of the image.

$\sigma=\sqrt{1 / N \sum\left(b_{i}-b\right)^{2}}$

Where $i=1,2,3 \ldots \ldots \ldots . . N$, b is the mean grey level value of the original image and bi is the grey level value of the surrounding region and $\mathrm{N}$ is the total number of pixel in the image.

\section{A REVIEW ON FEATURE EXTRACTION METHOD:}

Second stage is highlight extraction. An element extraction square recovers the most important highlights from the sign. These highlights will help the basic leadership instrument in giving the ideal yield. Highlight Extraction of EEG sign is a significant advance in any BCI based applications. This goes for depicting the EEG flag by (in a perfect world) a couple of important qualities called "highlights" Such highlights should catch the data implanted in EEG flag that is significant to portray the psychological states to distinguish, while dismissing the clamor and other non-pertinent data. All highlights removed are typically organized into a vector, known as an element vector. With BCI, there are 3 fundamental wellsprings of data that can be utilized to concentrate highlights from EEG signals:

> Spatial data: Such highlights would depict where (spatially) the pertinent sign originates from. By and by, this would mean choosing explicit EEG channels, or concentrating more on explicit channels than on some other. This adds up to concentrating on the sign starting from explicit territories of the mind.

> Spectral (frequential) data: Such highlights would portray how the power in some significant recurrence groups differs. Practically speaking, this implies the highlights will utilize the power in some particular recurrence groups.

> Temporal data: Such highlights would depict how the important sign fluctuates with time. By and by this implies utilizing the EEG sign qualities at various time focuses or in various time windows.

Published By: 
It involves

1. Discrete Cosine Transform (DCT)

2. Principle Component Analysis (PCA)

3. Hidden Markov Model (HMM)

4. Gaussian Mixture Model (GMM)

5. Discrete Wavelet Transform (DWT)

6. Lifting Scheme

7. K-mean clustering

According to the audit Discrete Wavelet Transform is usually utilized. Wavelets are valuable on the grounds that as you evacuate the most noteworthy frequencies, nearby data is held and the picture resembles a low goals rendition of the full pictures. Wavelets are numerical capacities that cut up information into various recurrence segments, and after that review every segment with a goals coordinated to its scale. These premise capacities are short waves with constrained span, in this manner the name 'wavelets' is utilized. The essential elements of the Wavelet Transform are scaled as for recurrence. The DWT is processed by progressive lowpass and highpass separating of the discrete time-area signal as appeared in figure 10.1 its noteworthiness is in the way it associates the consistent time multi-goals to discrete time channels. At every disintegration level, the half band channels produce sign crossing just a large portion of the recurrence band. This annihilation by 2 parts the time goals as the whole sign is presently spoken to by just a large portion of the quantity of tests. Along these lines, while the half band low pass sifting evacuates half of the frequencies and in this manner parts the goals, the destruction by 2 copies the scale. According to the survey Do and Vetterli presented the new two-dimensional contourlet change. This change is increasingly reasonable for building a multi-goals and multidirectional extension utilizing non-distinct pyramid directional channel keeps money with little excess factor. Contrasted and wavelet, contourlet give unique and adaptable number of bearings at each scale. It has been effectively utilized in picture upgrade, denoising and combination. Non sub-tested contourlet change deterioration is to figure the multi scale and distinctive course segments of the discrete pictures. It includes the two phases, for example, non-subsampled pyramid and non-subsampled directional channel bank to remove the surface, shapes and point by point coefficients.

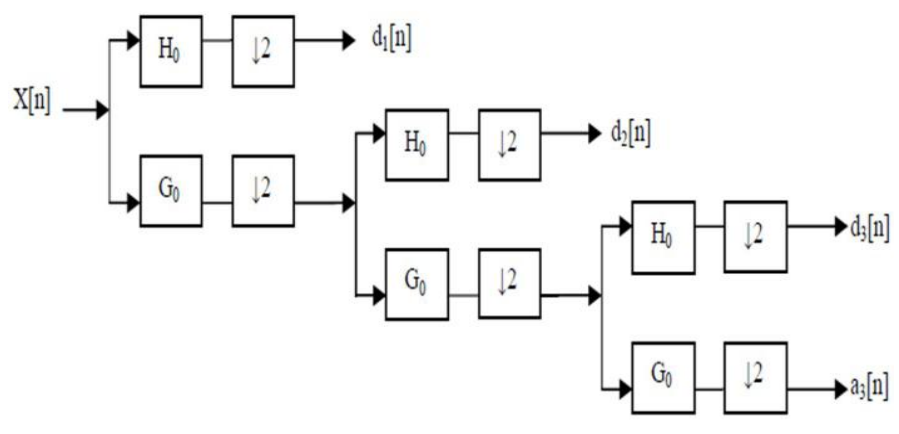

Fig.5 Three level wavelet decomposition tree
Table 10.1 subtleties the rundown of highlight. This examines techniques to acquire the pertinent qualities of cerebrum flag just as highlight determination strategies. The plan of an appropriate arrangement of highlights is a difficult issue. The data of enthusiasm for cerebrum sign is covered up in a profoundly loud condition, and mind sign include countless concurrent sources [10]. Cerebrum sign can be estimated through products channels. Not all data given by the deliberate channels is commonly pertinent for understanding the fundamental wonders of intrigue. Measurement decrease procedures, for example, head segment investigation or autonomous part examination can be connected to diminish the component of the first information, evacuating the insignificant and excess data. Computational expenses are accordingly diminished. A sign that might be of intrigue could be covered in reality by various sign from various cerebrum errands. Consequently, by and large, it isn't sufficient to utilize straightforward strategies. So in the Table 8.1 right off the bat, dimensional decrease strategies, for example, head part investigation or autonomous segment examination are clarified. Furthermore, time and additionally recurrence techniques, for example, coordinated sifting or wavelet changes, and parametric demonstrating, for example, autoregressive part, are likewise reviewed. Thirdly, a clarification is given of the normal spatial example calculation. This strategy structures a preprocessing spatial channel, by methods for spatial covariance from info information and sign's brightening that upgrades the contrast between classes before the component extraction arrange alongside its properties [12].

\section{CLASSIFICATION}

As indicated by the audit Artificial Neural Network (ANN) is progressively utilized for characterization while evacuating antiquities, a worldview that is identified with organic systems and attempts to emulate the structure of the human cerebrum. A neural system is a greatly comparable dispersed procedure, made up of straightforward handling units, which has a property for putting away information and making it accessible for use. A standout amongst the most significant properties of neural systems is their capacity to gain from models, that is, figure out how to create a specific yield when bolstered with a specific information.

The learning procedure includes alteration of the association loads, to cause its general execution to compare to an ideal execution characterized by the arrangement of preparing models. For every model in the preparation set there exists an info design and an ideal yield design. To prepare the system a model from the preparation set is picked, and bolstered to the system to perceive what yield it produces. On the off chance that the normal yield isn't acquired, the inward loads of the system is changed by some preparation calculation, in order to limit the distinction between the ideal and the real yield. The preparation is then proceeded with another preparation model, etc, until the system has achieved consistent state. Here a completely associated system is utilized and the standard back engendering calculation can be utilized for preparing. 
TABLE 10.1 SUMMARY OF FEATURE EXTRACTION

\begin{tabular}{|c|c|c|}
\hline \multirow{3}{*}{$\begin{array}{l}\text { Dimension } \\
\text { reduction }\end{array}$} & Method & Properties \\
\hline & PCA & $\begin{array}{l}>\text { Linear change } \\
>\text { Set of perhaps associated perceptions is changed into set of uncorrelated factors } \\
>\text { Optimal portrayal of information as far as negligible mean-square-blunder } \\
>\text { No ensures dependably a decent arrangement } \\
>\text { Valuable commotion and measurement decrease strategy. PCA necessitates that curios are uncorrelated } \\
\text { with the EEG signal }\end{array}$ \\
\hline & ICA & $\begin{array}{l}>\text { Splits a lot of blended sign into its sources } \\
>\text { Mutual factual freedom of fundamental sources is expected } \\
>\text { May degenerate the power range } \\
>\text { Powerful and vigorous instrument for antique evacuation. Ancient rarities are required to be } \\
\text { autonomous from the EEG signal }\end{array}$ \\
\hline Space & CSP & $\begin{array}{l}>\text { Spatial channel intended for 2-class issues. Multiclass expansions exist } \\
>\text { Good result for synchronous BCIs. Less successful for nonconcurrent BCIs } \\
>\text { Improved adaptations of CSP: WCSP, CSSP, CSSSP }\end{array}$ \\
\hline \multirow{4}{*}{$\begin{array}{l}\text { Time } \\
\text { Frequency }\end{array}$} & $\mathrm{AR}$ & $\begin{array}{l}>\text { Spectrum model } \\
>\text { High recurrence goals for brief time portions } \\
>\text { Not appropriate for non-stationary sign } \\
>\text { Adaptive form of AR: MVAAR }\end{array}$ \\
\hline & MF & $\begin{array}{l}>\text { Suitable for recognition of waveforms with steady fleeting attributes } \\
>\text { Its execution is influenced by the spatial goals. Some terminal area offer progressively discriminative } \\
\text { data for some particular mind exercises than other }\end{array}$ \\
\hline & CWT & $\begin{array}{l}>\text { Provides both recurrence and worldly data } \\
>\text { Appropriate for non-stationary sign }\end{array}$ \\
\hline & DWT & $\begin{array}{l}>\text { Provides both recurrence and worldly data } \\
>\text { Suitable for non-stationary sign } \\
>\text { Lessens the repetition and intricacy of CWT }\end{array}$ \\
\hline
\end{tabular}

For the system to prepare for preparing loads and one-sided ought to be instated. For preparing to begin a neural system a lot of information and target yield are required. During the learning procedure to diminish the system execution activity, the predispositions and loads are acclimated to the neural framework. The default capacity is the mean square mistake for feed forward neural system. All calculations use execution inclination capacity to discover changing loads to lessen execution. By performing calculations in reverse through the system utilizing back spread inclination is utilized.

Multilayered feed forward ANNs are having 2 distinct stages.

1. Learning stage

2. Execution arrange

In the learning stage ANN is prepared consistently on a particular arrangement of information to take explicit information and send the particular yield. While $\mathrm{n}$ the execution stage ANN will return explicit yield contingent upon the info. The working of feed forward ANN is as per the following: When the information is given to info layers, it goes through all layers until it achieves the yield layer. Where yield is given, it's anything but difficult to assess the yield going through the system in the feed forward ANN.

point it's hard to locate the unmistakable yield. For non-time subordinate issues feed forward system is the best. Multilayer feed forward ANN appeared in which neurons of each layer are interface with neuron of other layer, which is known as completely associated system.

\section{REVIEW ON COMBINATION OF WAVELET DECOMPOSITION, ICA AND THRESHOLDING METHOD FOR REMOVAL OF ARTIFACTS IN EEG SIGNALS}

So as to expel relics from an EEG flag there is an another strategy which is a blend of wavelet disintegration, Independent Component Analysis (ICA) and thresholding. This technique can decrease or evacuate the ancient rarities types including squints, ECG, EOG and EMG. This strategy can work on just 16 EEG channels. What's more, it doesn't require any extra flag. Contrasted with existing technique one which required the huge number of terminal channels likewise a few references signals. So this strategy works on a generally modest number of EEG channels.

Steps includes in this strategy:Right off the bat this technique endeavors to expel ancient rarity parts from $1 \mathrm{~s}$ windows of the EEG by means of the accompanying advances.

1) Decompose the EEG on each channel into a lot of estimate and detail coefficients by means of a wavelet disintegration. It is signified by $c_{-} j \wedge i \in C$ the $j$ th coefficient set from the arrangement of all coefficients $\mathrm{C}$, from channel $\mathrm{I}$.

2) Groups the all coefficients at a similar decay level from each channel into sets of coefficients, $A \_n=$ $c_{-} \mathrm{j}^{\wedge} \mathrm{i} \in \mathrm{C} \mid \forall \_\mathrm{i} \in \mathrm{K}, \mathrm{j}=\mathrm{n}$, where $\mathrm{K}$ is the arrangement of channels and $n$ means the deterioration level.

3) For the arrangement of guess coefficients (A_1) gauges an ICA de-blending grid to isolate the coefficients into maximally measurably free parts (ICs).

4) Multiplies the arrangement of guess coefficients by the demixing grid.

5) Identify ICs which contain curios and expel them.

6) Invert the ICA deterioration to get a gauge of the cleaned estimation coefficient set $\mathrm{A}_{-}^{-} 1$.

7) Identifies spike zones in both the guess and detail coefficient sets and afterward applying the delicate thresholding so as to diminish their size.

8) Reconstructs the cleaned EEG from the wavelet guess and detail coefficient sets.

Wavelet deterioration: Wavelets endeavor to break down a sign by convolving it with a mother wavelet work at a scope of various time and recurrence areas and estimating the quality of the sign as a coefficient of the wavelet work. 
Here the Discrete Wavelet Transform (DWT) is utilized; this scales to the sign at a discrete arrangement of times and frequencies. The Symlet 4 'Sym4' is a mother wavelet utilized in this work to decay the sign into estimation and detail coefficients down to 2 deterioration levels.

Autonomous part investigation: Independent segment examination (ICA) id used to isolate the multivariate sign into subcomponents which are maximally measurably free from each other. The EEG is accepted to emerge from the summed electrical action created from various autonomous sources. ICA endeavors to gauge the blending procedure which offered ascend to the EEG from these sources and afterward, by reversing the blending framework, to endeavor to reproduce the sources.

Recognizable proof of curio sullied ICs: The ICs which contain antiquities should be distinguished and expelled. Various methodologies are there to distinguish. This technique expects to evacuate eye squints, EOG action identified with other (non-flicker) eye developments, EMG action identified with muscle developments, and electrocardiogram (ECG) ancient rarities. So ICs containing every one of these antiques might be recognized in a different manner. Ancient rarities are known to vary from clean EEG subsequently it had the accompanying properties.

1) There is a measure of transient reliance inside the sign.

2) The measure of spiking movement is seen inside the sign.

3) The similitude of the power otherworldly thickness conveyance of the watched EEG to a $1 / \mathrm{F}$ appropriation. Where F signifying recurrence.

4) The kurtosis estimation of the sign estimating peakedness of the sign amplitudes after some time.

5) The power ghostly thickness in the Gamma recurrence band or more $(>30 \mathrm{~Hz})$.

6) The pinnacle amplitudes of the EEG time arrangement.

7) The standard deviation and topographic appropriation of the plentifulness estimations of the EEG time arrangement.

ICs are recognized as liable to contain a curio when they surpass edges for at least one of these criteria. Notwithstanding, there is a probability for a time of clean EEG to surpass an edge that implies a bogus positive distinguishing proof. In this way, to limit the impact of such false positive ancient rarity location the quantity of edges surpassed by every IC is tallied. ICs which surpass multiple edges are evacuated [31].

So among every one of the strategies precision of the mix of wavelet disintegration, free part investigation (ICA) and thresholding strategy yields better execution contrasted with other existing methods.

\section{CONCLUSION}

EEG is developed for use in BCI. EEG signals help us to diagnose the mental states and various neurological diseases. The EEG signals are very subjective, non-correlated, non-Gaussian, random in nature. According to the review, a lot of improvement needs to be done in order to obtain the cleaned EEG signals. Different techniques are introduced to get a cleaned EEG signals by removing artifacts contaminated by it. This article details the fundamental aspects of the BCI system and artifacts removal techniques. Among all the techniques accuracy of the combination of wavelet decomposition, independent component analysis (ICA) and thresholding method yields better performance compared to other existing techniques.

\section{REFERENCES}

1. Foud Amin and Mohamed mostafafouad, "Cerebrum Computer Interface: A Review", 2015.

2. J. R.Wolpaw, N. Birbaumer, D. J. McFarland, G. Pfurtscheller, and T. M. Vaughan, "Cerebrum PC interfaces for correspondence and control." ClinNeurophysiol, vol. 113, pp. 767-791, Jun. 2002.

3. A. Kubler, F. Nijboer, J. Mellinger, T. M. Vaughan, H. Pawelzik, G. Schalk, D. J. McFarland, N. Birbaumer, and J. R. Wolpaw, "Patients with ALS can utilize sensorimotor rhythms to work a cerebrum PC interface." Neurology, vol. 64, no. 10, pp. 1775-7, May 2005.

4. Ian Daly, Reinhold Scherer, Martin Billinger, and GernotM"ullerPutz, "Power for cerebrum PC interface" 2015.

5. Sebastian Halder and Wolfgang Rosenstiel,"online ancient rarity evacuation for Brain-Computer Interface utilizing bolster vector machine" IEEE Aug 2007.

6. Luis Fernando Nicolas-Alonso and "Mind Computer Interfacing" Jan 2012.

7. H. Nolan, R. Whelan, and R. B. Reilly, "Quicker: Fully Automated Statistical Thresholding for EEG antiquity Rejection." Journal of neuroscience strategies, vol. 192, no. 1, pp. 152-62, Sep. 2010.

8. I. Daly, N. Nicolaou, S. J. Nasuto, and K. Warwick, "Computerized antiquity expulsion from the electroencephalogram: a near report." Clinical EEG and neuroscience, vol. 44, no. 4, pp. 291306, Oct.2013.

9. P. S. Kumar, R. Arumuganathan, K. Sivakuma, and C. Vimal, "Expulsion of visual antiquities in the EEG through wavelet change without utilizing an EOG reference channel," vol. 1, no. 3, pp. 188-200, 2008.

10. Veer Amol Motinath, Chandan Kumar and Maheshkumar H Kolekar "A Novel ECG Data Compression Algorithm utilizing Best Mother Wavelet Selection", Sept 2016.

11. Dapeng W U, Boran Yang and Honggang Wang," An Energy-Efficient Data Forwarding Strategy for heterogeneous WBANS" Sept 2016.

12. Hyohyeong Kang, Yunjun Nam, and Seungjin Choi, " Composite Common Spatial Pattern for Subject-to-Subject Transfer," IEEE sign handling letters, vol. 16, no. 8, Aug 2009.

13. Fabien Lotte, and Cuntai Guan, "Regularizing Common Spatial Patterns to Improve BCI Designs: Unified Theory and New Algorithms" Biomedical building, vol. 58, no. 2, Feb 2011.

14. Eugene Teo, Alvin Huang, and $\mathrm{Li}$ Haihong Zhang, "Media Communication Center Using Brain Computer Interface",2006

15. Akaysha C. Tang, Matthew T. Sutherland, an and Christopher J. McKinneya, "Approval of SOBI parts from high-thickness EEG", Jan 2005.

16. I.I. Goncharova, D.J. McFarland, T.M. Vaughan, J.R. Wolpaw, "EMG pollution of EEG: unearthly and geological qualities", March 2003.

17. Joseph T. Gwin, Klaus Gramann, Scott Makeig and Daniel P. Ferris, "Expulsion of Movement Artifact From High-Density EEG Recorded During Walking and Running", 2010.

18. Hossein Mamaghanian, "Packed Sensing for Real-Time Energy-Efficient ECG Compression on Wireless Body Sensor Nodes", Sept 2011

19. X. Yong, M. Fatourechi, R. K. Ward, and G. E. Birch, "Programmed ancient rarity recognition in a self-managed cerebrum PC interface framework," aug2011.

20. S. Fitzgibbon, D. Forces, K. Pope, and C. Clark, "Evacuation of EEG commotion and antique utilizing visually impaired source partition." Journal of clinical neurophysiology : official production of the American Electroencephalographic Society, vol. 24, no. 3, pp. 232-243, Jun. 2007.

21. T. Radüntza, J. Scoutena, O. Hochmuthb, B. Meffertb, "EEG ancient rarity end by extraction of ICA-segment highlights utilizing picture preparing calculations" 2015.

22. Repovas, G "Managing commotion in EEG recording and information investigation" 2010.

23. Semmlow, J. "Biosignal and Medical Image Processing" 2011.

24. Palaniappan, R, Syan, C.S, Paramesran, R "Current Practices in Electroencephalogram-Based Brain-Computer interfaces" vol. II, second edn. IGI Global, Hershey, pp. 888-901, 2009. 
25. [25] Mihajlović, V, Garcia-Molina, G, Peuscher, J "Dry and water-based EEG terminals in SSVEP-based BCI applications" 2013.

26. Hazrati, M.K, Husin, H.M, Hofmann, U.G "Remote mind signal chronicles dependent on capacitive anodes" 2013.

27. Lepola P, Myllymaa, S, Toyras, J, Mervaala, E, Lappalainen, R, Myllymaa, K "Protected plan of screen-printed EEG cathode set decreases obstruction get" 2014.

28. T. Elbert, W. Lutzenberger, B. Rockstroh, and N. Birbaumer, "Expulsion of visual relics from the EEG - a biophysical way to deal with the EOG," vol. 60, no. 5, pp. 455-463, 1985.

29. R. J. Croft and R. J. Barry "Expulsion of visual relic from the EEG: a review,",pp. 5-19, 2000.

30. Guangyu, B, Xiaorong, G, Yijun, W Bo, H. Shangkai G, "VEP-based brain-computer interfaces: Time, frequency, and code modulations", 2009.

31. Ian Daly, Reinhold Scherer and Gernot Muller-Puts, "Power: Fully Online and mechanized antiques Removal for mind Computer interfacing", 2013.

32. Reinhold Scherer, Member, IEEE, Günter Moitzi, Ian Daly, and Gernot R. Müller-Putz," On the Use of Games for Noninvasive EEG-Based Functional Brain Mapping", JUNE 2013.

33. M. K. Islam, A. Rastegarnia, A. T. Nguyen, and Z. Yang, Artifact Characterization and Removal for In-Vivo Neural Recording, Journal of Neuroscience Methods, (2014), Vol.226, pp.110-123.

34. M. K. Islam, A. Rastegarnia, and Z. Yang, A Wavelet-Based Artifact Reduction from Scalp EEG for Epileptic Seizure Detection, Biomedical and Health Informatics, (2015), Vol.99, pp.1-12.

35. Chang-Young Jung, Sakala Satya Saikiran , "A survey on EEG antiques and its distinctive expulsion system " 2016.

36. Erin Hecht and Dietrich Stout, "Methods for Studying Brain Structure and Function".

37. Carrie A. Joyce, Irina F. Gorodnitsky and Marta Kutas, "Programmed expulsion of eye development and squint ancient rarities from EEG information utilizing blind segment detachment".

38. Hallez H,De Vos M, Vanrumste B, Van Hese P, Assecondi S and VanLaere K P, et al."Removing muscle and eyes antiquities utilizing visually impaired source division methods in icyal EEG source imaging Clin Neurophysiol",2009.

39. Klados M A, Papadelis C ,Braun C, Bamidis P D.Reg-ica: a half breed strategy joining visually impaired source division and regres-sion strategies for the dismissal of visual curios. Biomed Signal Process Control 2011;6:291-300.

40. Ng SC, Raveendran P,"Enhanced beat extraction utilizing visually impaired source partition and wavelet change". IEEE Trans BiomedEng 2009;56:2024-34.

41. Cohen MX, "Breaking down neural time arrangement information: hypothesis and practice", MIT Press; 2014. p. 51-4.

42. Turnip A. "Programmed curios expulsion of EEG sign utilizing vigorous head part examination". In: Technology, Informatics, Management, Engineering, and Environment (TIME-E), 2014 second International Conference. 2014. p. 331—4.

43. Turnip A, Junaidi E. "Expulsion antiquities from EEG sign utilizing free segment investigation and head segment examination". In: Technology, Informatics, Management, Engi-neering, and Environment (TIME-E), 2014 second International Conference. 2014. p. $296-302$.

44. Flexer A, Bauer H,Pripfi J,Dorffner G,Using ICA for evacuation of visual antiques in EEG recorded from visually impaired subjects",2005.

45. Klados MA, Papadelis C, Braun C, Bamidis P D."Reg-ica:ahybrid procedure consolidating visually impaired source division and relapse systems for the dismissal of visual ancient rarities". Biomed Signal Process Control 2011;6:291-300.

46. Zhao Q, Hu B, Shi Y, Li Y, Moore P, Sun M, et al. "Programmed identification and expulsion of visual antiquities in EEG - improved versatile indicator filtering for compact applications. IEEE Trans Nanobioscience "2014.

47. De Vos M, Deburchgraeve W, Cherian P, Matic V, SwarteR, Govaert $\mathrm{P}$, et al. "Computerized antiquity expulsion as preprocessing refines neonatal seizure location". Clin Neu-rophysiol 2011;122:2345-54.

48. Ma J, Bayram S, Tao P, Svetnik V. "High-throughput visual relic decrease in multichannel EEG utilizing part subspace projection" J Neurosci Methods2011;196:131—40.

49. Ma J, Bayram S, Tao P, Svetnik V." Muscle relics in multi-channel EEG: attributes and decrease". Clin Neurophysiol2012;123:1676—86.

50. Kierkels JJ, Riani J, Bergmans JW, Van Boxtel GJ. "Utilizing an eye tracker for precise eye development antiquity rectification". IEEE Trans Biomed Eng 2007;54:1256-67.

51. Noureddin B, Lawrence P D, Birch GE. "Online expulsion of eye development and flicker EEG antiques utilizing a fast eyetracker". IEEE Trans Biomed Eng 2012;59:2103-10.

52. Savelainen A." Movement relic recognition from electroencephalogram using accelerometer". Ace's theory. Aalto University School of Science and Technology; 2011.

53. Sweeney K." Motion relic handling strategies for physiological sign". PhD proposal. National University of Irel and Maynooth; 2013.

54. O'Regan S, Faul S, Marnane W. "Programmed recognition of EEGartefacts emerging from head developments utilizing EEG and gyrator signals". Medications Eng Phys 2013;35:867-74.

55. Mihajlovic V, Li H, Grundlehner B, Penders J, SchoutenA. "Examining the effect of power and developments on impedance extent and EEG". In: Engineering in Medicine and Biology Society (EMBC), 2013 35th Annual International Conference of the IEEE. 2013. p. $1466-9$.

56. Mihajlovic V, Patki S, Grundlehner B. "The effect of head developments on EEG and contact impedance: an adaptivefiltering answer for movement curio decrease". In: Engineering in Medicine and Biology Society (EMBC), 201436th Annual International Conference of the IEEE. 2014.p. 5064-7.

57. Gwin JT, Gramann K, Makeig S, Ferris DP. "Expulsion of development curio from high-thickness EEG recorded during strolling and running". J Neurophysiol 2010;103:3526—34. 